\title{
Ozone-based eye drops in anterior segment pathologies: rationale and pre-clinical data
}

\author{
Andrea Marchegiani, Andrea Spaterna \\ School of Biosciences and Veterinary Medicine, University Hospital of Educational Veterinary Sciences, University of \\ Camerino, Italy
}

\begin{abstract}
Inflammatory anterior segment diseases necessitating adequate antinflammatory therapy as conjunctivitis, keratoconjunctivitis sicca and corneal ulcers are common eye disorders encountered both in humans and in animals, actually treated by topical administration of eye drops containing antinflammatory and antibacterial agents. The current molecules often lack efficacy because infections in hypoxic tissue contain methicillin-resistant Staphylococcus aureus and Pseudomonas aeruginosa and new products for the treatment of ocular pain and inflammation are need. The use of ozone in certain anterior segment pathologies could be providential due to anti-inflammatory and bactericidal activity, in addition to promoting tissue repair properties. Ozonated oils have the same properties of gaseous ozone and are well tolerated by tissues. In the present study ozone-based eye
\end{abstract}

Correspondence: Andrea Marchegiani, School of Biosciences and Veterinary Medicine, University Hospital of Educational Veterinary Sciences, University of Camerino, via Circonvallazione 93/95, 62024 Matelica (MC), Italy.

Tel: $+39.0737403403-+39.0737401709$

Email: andrea.marchegiani@unicam.it

Key words: Ozone; Ozone-based eye drop; Anterior segment; Conjunctivitis; Keratitis.

Contributions: the authors contributed equally.

Conflict of interest: the authors declare no potential conflict of interest.

Conference presentation: part of this paper was presented at the $20^{\text {th }}$

AICCER Congress, 2011 March 9-11, Rimini, Italy.

Acknowledgments: the authors would like to thank SIOOT, Scientific Society of Oxygen-Ozone Therapy, Gorle (BG), Italy.

Received for publication: 12 April 2017.

Accepted for publication: 12 April 2017.

(C) Copyright A. Marchegiani and A. Spaterna, 2017

Licensee PAGEPress, Italy

Ozone Therapy 2017; 2:6743

doi:10.4081/ozone.2017.6743

This article is distributed under the terms of the Creative Commons Attribution Noncommercial License (by-nc 4.0) which permits any noncommercial use, distribution, and reproduction in any medium, provided the original author(s) and source are credited. drops were used to treat recurrent conjunctivitis and keratitis with excellent results in terms of pathology resolutions and satisfaction of the owners.

\section{Introduction}

Inflammatory anterior segment diseases requiring adequate antinflammatory therapy as conjunctivitis, keratoconjunctivitis sicca and corneal ulcers are the most common eye disorders encountered in animals, ${ }^{1}$ and share with human some symptoms as redness, chemosis, and exudation. Accordingly, the topical administration of eye drops containing antinflammatory and antibacterial agents is often prescribed. A topical dosage of one or two drops of collyrium every 4 hours over 3 to 7 days is recommended, even though to achieve clinical resolution multiple and frequent instillation are often required, and some patients tend to become uncompliant to frequent medications. ${ }^{1,2}$

The current agents are quite expensive and often they lack efficacy because infections in hypoxic tissue contain methicillin-resistant Staphylococcus aureus and Pseudomonas aeruginosa; in some cases, they are inadequate and can cause unacceptable side effects.

In particular, there is a need for new products for the treatment of ocular pain and inflammation, such as during external ocular infections and inflammations, due to the related risk of blindness.

The overuse of antibiotics in the treatment of infectious diseases, and the appearance of multi-drug resistant bacterial strains, has driven research towards the study of antimicrobial agents from essential oils. ${ }^{3}$

The use of ozone $\left(\mathrm{O}_{3}\right)$ in certain ocular pathologies such as the ones of anterior segment could be providential due to the ozone properties. Ozonated oils have the same properties of gaseous ozone, are well tolerated by biological tissues and their biological activities are related to oxygenated compounds. ${ }^{3}$ They are able to eliminate the pathogens by direct oxidation mediated by hydrogen peroxide and lipoperoxyde, and selective cytotoxicity on fastdividing cells, through bacterial lysis and cell death, negative regulation on mitochondrial activity in bacteria and disturbance on virus lytic enzimes activity, in a more superimposable manner than that of phagocytic cells of the immune system. ${ }^{4}$

Moreover, ozone promotes wound healing by releasing oxygen $\left(\mathrm{O}_{2}\right)$, platelet-derived growth factor (PDGF) and transforming grow factor $\beta$ (TGF- $\beta$ ), thus activating the proliferation of fibroblasts and successive healing.

In addition, ozone promotes cells adaptation to oxidative stress and attenuates pathophysiological events mediated by reactive oxygen species. Ozone oxidative preconditioning significant- 
ly decreased malondialdehyde levels and increased the activity of Superoxide Dismutase, Glutathione peroxidase and Catalase. Serum interleukin-1 beta levels tend to decrease with ozone oxidative preconditioning. ${ }^{4}$

\section{Aim of the study}

Based on the many features and possible applications of ozone, aims of the present study was a preliminary evaluation of repair and regeneration effect of ozonated oil (Ozodrop ${ }^{\circledR}$; FB Vision, Ascoli Piceno, Italy) instilled three times a day in external ocular spontaneous pathologies in animals.

\section{Materials and Methods}

A 26-year-old, neutered male, Italian saddle horse, was conducted to the Veterinary Teaching Hospital of University of Camerino because of right eye exophthalmos due to retrobulbar neoformation (probably neoplasm, osteosarcoma) and recurrent conjunctivitis, treated with topical antibiotic (tobramycin) and both topical and systemic NSAID (piroxicam plus flunixine meglumine) without any significant improvement. The day of presentation the horse showed a normal left eye; regarding right eye, these were the symptoms present: blepharitis and blepharospasmus, edematous and hyperemic conjunctiva, plenty mucous ocular discharge (Figure 1). The day after the beginning of the therapy, blepharospasmus disappeared, and a reduction in blepharitis and conjunctival edema was noted. Moreover, there was a slight reduction in ocular discharge. After three days of therapy blepharitis and conjunctivitis disappeared and after a week of therapy the eye became completely normal (Figure 2).

A 6-month-old male European short hair cat (Figure 3) was affected by chronic conjunctivitis present from birth, unresponsive to the traditional antimicrobic and antinflammatory therapy. The day of presentation the patient showed marked conjunctival edema, especially in right eye, reddening of the conjunctiva and mucous discharge on both eyes. Conjunctival bacteriological swabs revealed normal bacteria count in right eye ( $20 \mathrm{cfu})$ and the presence of Sthapilococcus spp. and Enterococcus spp.; in left eye

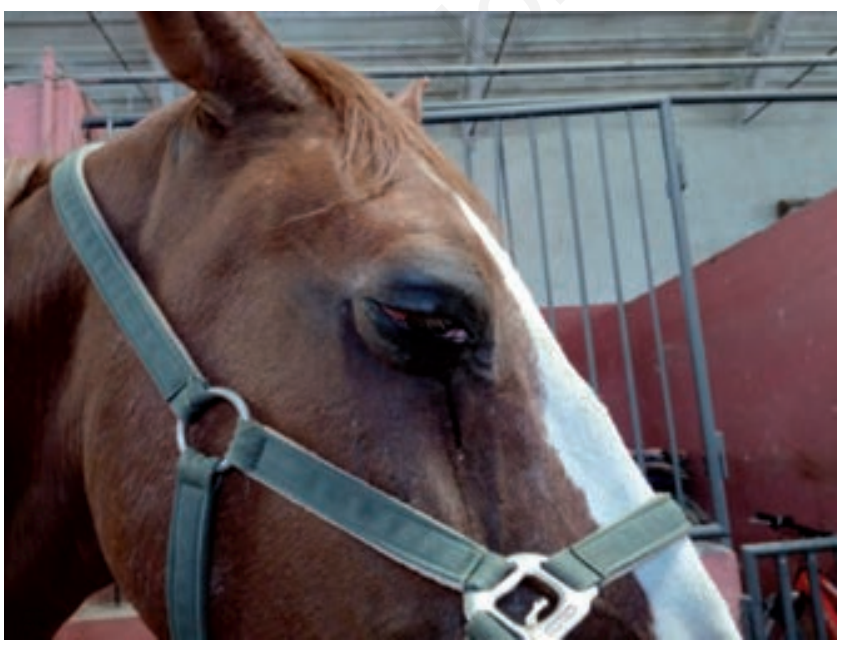

Figure 1. Right eye of 26-year-old horse before starting the treatment. Note blepharitis and blepharospasmus, edematous and hyperemic conjunctiva, plenty mucous ocular discharge. was detected an increased amount of Sthapilococcus spp. (140 cfu). Therapy was consistent with instillation of one eye drop of collyrium in both eyes, twice a day until clinical and bacteriological resolution. After three days of therapy conjunctival bacterial count became normal in both eyes (20 cfu) and Enterococcus spp. disappeared. In ten days of therapy conjunctival symptoms were no more detectable (Figure 4).

A 3-year-old male English Bulldog (Figure 5) was referred due to the presence of chronic keratitis in the right eye due to entropion of both eyelids. Because of other problems not related to the presenting pathologies, it has not been possible to operate the dog immediately due to the high risk of general anaesthesia and in according with the owner it was decided to begin topical therapy with instillation of one drop of collyrium twice a day until surgery. The day of the presentation the dog showed keratitis, corneal edema and deep and superficial neovascularization. Discomfort, pruritus and ocular mucous discharge were also present. After ten days of therapy, keratitis were almost disappeared, corneal edema has resolved and neovascularization remains only with a single, small calliper vessel arising from limbus at the level of medial cantus (Figure 6). Entropion and mucous discharge were still present.

\section{Results and Discussion}

Conjunctivitis in horses represent a therapeutic challenge because they require different active principles to be administered,

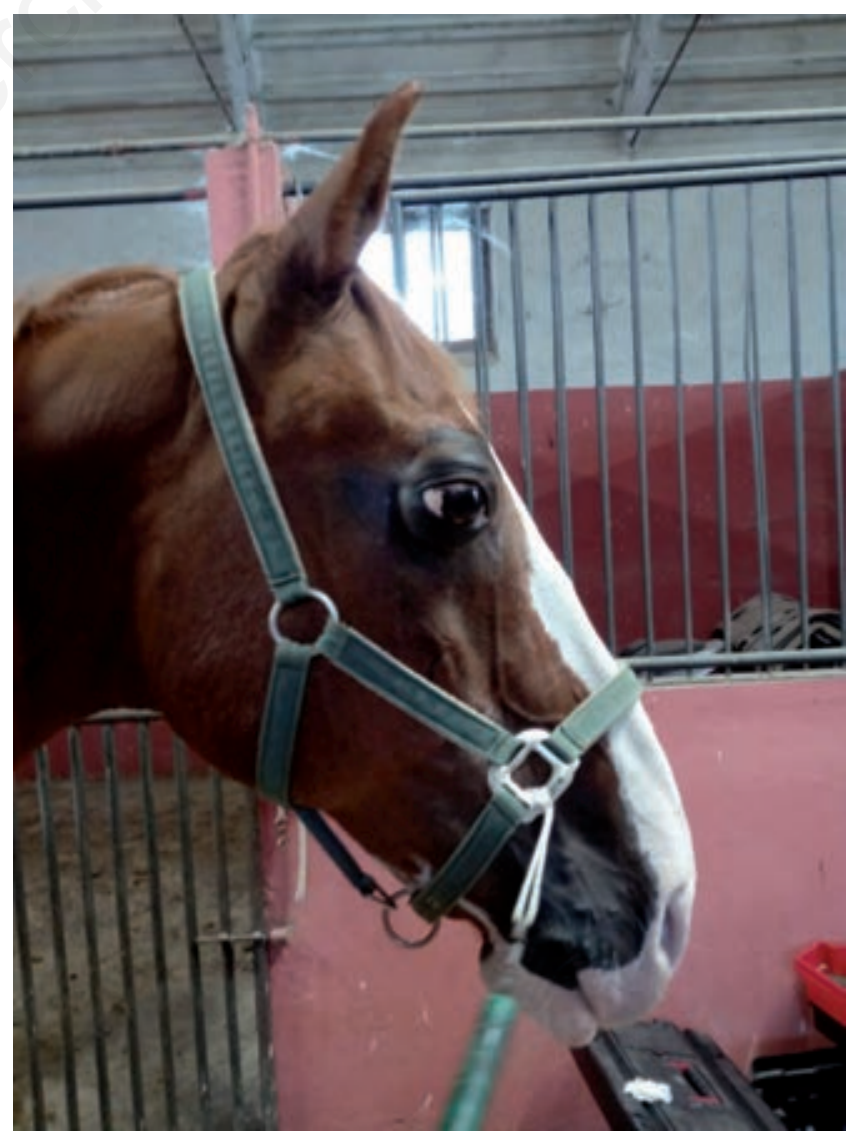

Figure 2. Same eye of Figure 1 after seven days of therapy. Normal eye. 
almost never present a single eye-drops and horses tend to get uncooperative in a little time, so often the veterinarian have to resort to alternative methods of treatment (i.e.: sub-palpebral lavage systems).

In this case of recurrent conjunctivitis secondary to retrobulbar lesion, the ozone-based eye drops were effective in solving the problem. The frequency of three times daily was well tolerated by the animal that has shown good compliance to treatment and longlasting therapy results.

Conjunctivitis in cats represents a common problem and traditional therapy involves the use of topical antibiotics and NSAIDs in some cases even systemic.

In this case the ozone-based eye drops was able to reduce the congestion of conjunctiva in ten days of application. Moreover, after only three days of treatment Enterococcus spp., it was no longer detected and total bacteria count has decreased to levels considered normal in cats.

Entropion represent a common problem in brachycephalic dogs and cornea is often affected by entropion-related changes such as erosion/ulceration, inflammation, neovascularization, pigmentation. Entropion management is usually surgical and requires general anaesthesia.

In cases as the present one, where surgery cannot be quickly performed, ozone-based eye drops were able to reduce discomfort of the dog and improve corneal health, despite the persistence of underlying cause (mechanical clutch of the hairs of the lids on the cornea).

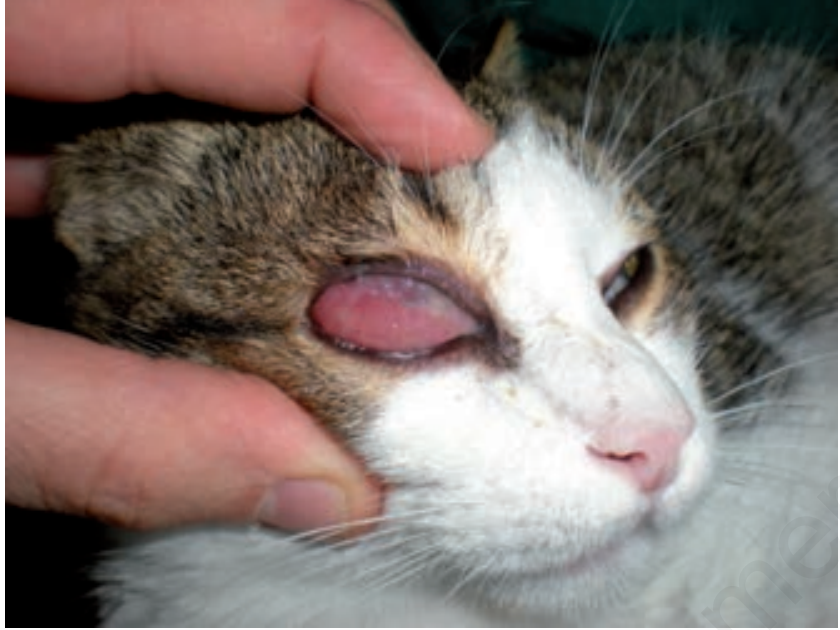

Figure 3. Right eye of 6-months-old cat before starting the treatment. Note marked conjunctival edema, reddening of the conjunctiva and mucous discharge.

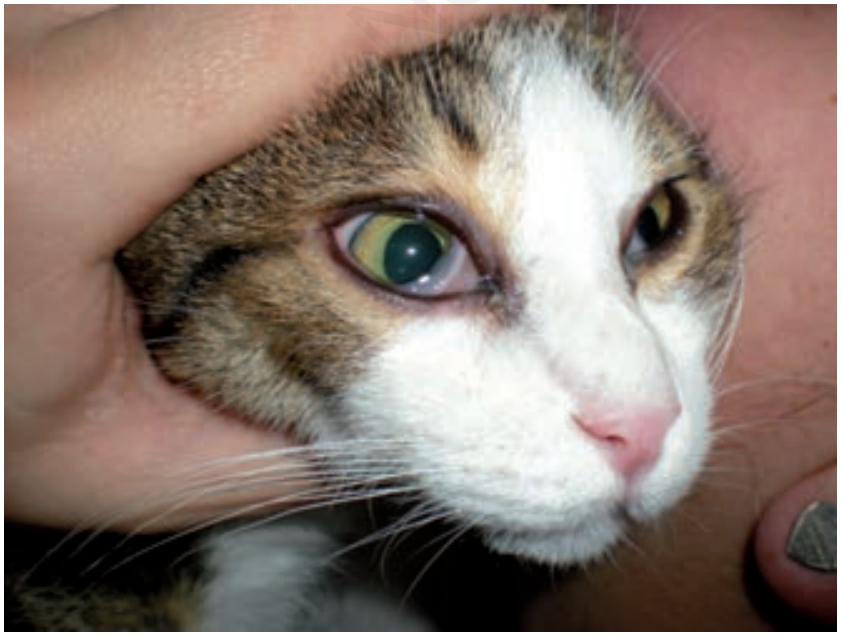

Figure 4. Same eye of Figure 3 after ten days of therapy. Normal eye, mucus discharge still present.

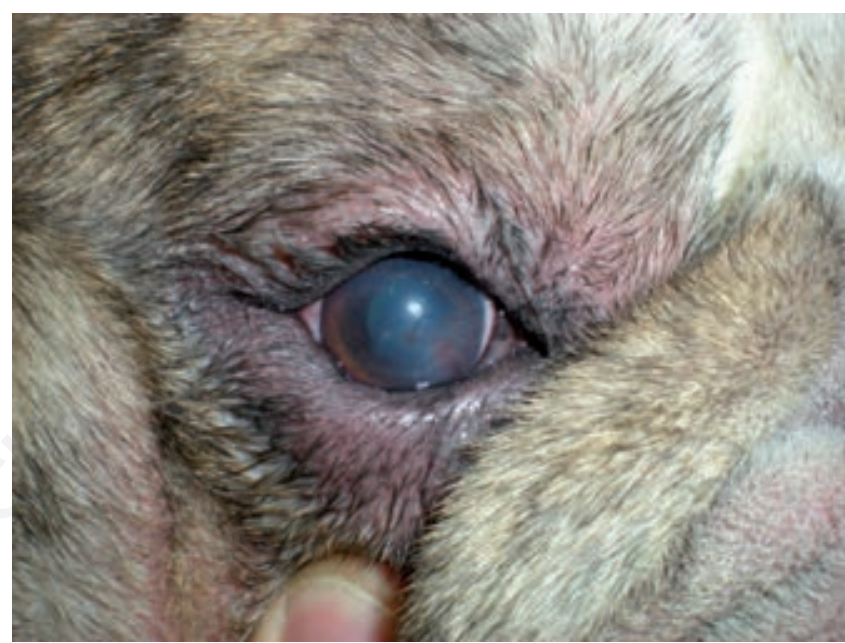

Figure 5. Right eye of 3-years-old English bulldog before starting the treatment. Note entropion, mucous discharge, keratitis, corneal edema, deep and superficial neovascularization.

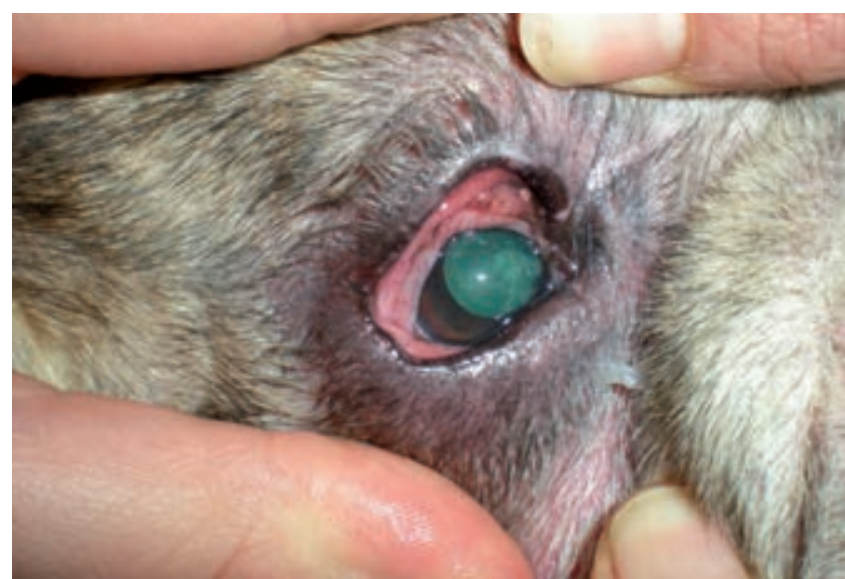

Figure 6. Same eye of Figure 5 after ten days of therapy. Corneal edema has resolved and neovascularization remains only with a single, small caliper vessel arising from limbus at the level of medial cantus. Entropion and mucous discharge are still present. 


\section{Conclusions}

This preliminary in vivo study has demonstrated as ozonebased eye drops has anti-inflammatory and bactericidal activity, in addition to promoting tissue repair. All of these beneficial and therapeutic effects are contained in a unique ocular preparation, reducing the risk of un-cooperation of patients in receiving medications. Ozone-based eye drops are very attractive by their versatility and various potential uses in ophthalmology in animals and humans. In conclusion, from these preliminary results, ozone-based eye drops represent a valid and suitable alternative therapy for the management of external ocular pathologies in animals and, given its antimicrobial properties, could be used as an aid for the preparation of the operative field for intra- and extra-ocular surgical procedures (i.e. cataract surgery or vitreal injection).

\section{References}

1. Hendrix DVH. Diseases and Surgery of the Canine Conjunctiva and Nictitating Membrane. In: Kirk N. Gelatt, ed. Veterinary Ophthalmology. 4th ed., Hoboken, New Jersey, NJ: Blackwell Publishing; 2007, pp 355-437.

2. Baeyens V, Felt-Baeyens O, Rougier S et al. Clinical evaluation of bioahdesive opthalmic drug insert (BODI) for the treatment of external ocular infections in dogs. JCR, 2002;85:163-8.

3. Sechi LA, Lezcano I, Nunez $\mathrm{N}$ et al. Antibacterial activity of ozonized sun-ower oil (Oleozon). JAM, 2001;90:279-84.

4. Bardi JS. News and Views; The Scripps Research Institute 2002. 